\title{
A Transformer Replacement Decision Method Based on Probability Assessment of Failure Rate
}

\author{
Gang Liang', Shengwei $\mathrm{Li}^{2}$, Yan $\mathrm{Qi}^{3}$, Jing Cao ${ }^{3}$, Yi Hao ${ }^{4}$, Wenfu Chen ${ }^{4}$ \\ ${ }^{1}$ State Grid Tianjin Dongli Electric Power Supply Company, Tianjin, China \\ ${ }^{2}$ State Grid Tianjin Economic Research Institute, Tianjin, China \\ ${ }^{3}$ Tianjin Electric Power Research Institute, Tianjin, China \\ ${ }^{4}$ State Grid Tianjin Dispatching and Control Center, Tianjin, China \\ Email: *youjianweiyang17@163.com
}

How to cite this paper: Liang, G., Li, S.W., Qi, Y., Cao, J., Hao, Y. and Chen, W.F. (2017) A Transformer Replacement Decision Method Based on Probability Assessment of Failure Rate. Energy and Power Engineering, 9, 748-755.

https://doi.org/10.4236/epe.2017.94B080

Received: April 4, 2017

Accepted: April 10, 2017

Published: April 17, 2017

\begin{abstract}
Reasonable probability assessment of transformer failure rate (FR) is a critical reference to the transformer replacement work. At present, the lack of support theory for transformer replacement usually causes reliability and economy issues for power companies. For this reason, a transformer replacement decision method based on probability assessment of FR is proposed. Firstly, a first order model of transformer paper degradation is proposed. Then, the Weibull Distribution is used by Monte Carlo Simulation (MCS) to generate the variations of Degree of Polymerization (DP) along with time based on the historical data, and the transformer FR is determined. When the FR is higher than a pre-defined threshold value, the transformer should be replaced for reliability purpose. Finally, the effectiveness of the proposed method for the transformer replacement decision is verified by a typical engineering application.
\end{abstract}

\section{Keywords}

Transformer Replacement, Probability Assessment, Weibull Distribution, Degree of Polymerization

\section{Introduction}

The safe operation of power transformer is one of the important defenses to the reliability operation of power system. In order to ensure the transformer always works under a healthy operation status, it is critical to assess the failure rate (FR) of transformer and replace the old on eat a most suitable time [1]. As one of the most important equipment in power system, the transformer with a large num- 
ber accounts for a significant proportion of the total cost of power companies. As a result, determining a reasonable replacement time for the transformer can effectively reduce the cost of power companies.

At present, there usually exists two extremes in the replacement of transformer. On the one side, the old transformer in service always working exceeds its service life limitation, which may lead to a high maintenance cost. On the other side, frequently replace transformer in pursuit of the high performance of new transformers will definitely results in unnecessary waste. The old transformer still occupies a large proportion in power system at the present time, and the replacement work is very urgent. Therefore, considering the relationship between the healthy status and service life of transformer, it is critical to determine a transformer replacement decision method for providing the scientific basis for the power enterprises to carry out technology reconstruction of power transformer.

A lot of studies have already been carried out in this area. In refs [2] [3], the method of transformer service life assessment based on large data analysis was proposed. In [4], a transformer life assessment method based on fuzzy theory and markov chain method was developed considering the transformer performance decline process. A transformer insulation life determination method based on insulation equivalent aging model was proposed in [5]. A method for transformer life assessment based on neural network was proposed in [6] [7]. A transformer state estimation method based on risk analysis method was developed in [8] [9], in order to predict the service life of a transformer. In above researches, the service life of the transformer is assessed from different aspects. However, various kinds of uncertainties exist in the operation process of the transformer, which will seriously impact the security operation of the transformer. As a result, there is always a contradiction between economic and reliability, when decide whether to replace a transformer or not. Therefore, consider the influences from many uncertainties, it is of great practical value to develop adecision method for transformer replacement, in order to guide the transformer technical transformation process.

For this reason, a transformer replacement decision method based on probability assessment of FR is proposed. A first order model of transformer paper degradation is used to depict the mathematical relationship between service life and FR, and the Weibull Distribution is used to generate the variations of Degree of Polymerization (DP) by Monte Carlo Simulation (MCS) along with time. The transformer FR is then determined. When the FR is higher than a pre-defined threshold value, the transformer should be replaced for high reliability purpose.

\section{Transformer Aging Mechanism Modeling}

Transformer is composed of iron core, high and low voltage windings, major insulation, dielectric oil, oil tank, and drive pipe, etc. According to the refs [10] [11], the insulation paper is the weakest part related to the transformer reliabili- 
ty. The aging process of insulation paper can reflect the aging process of transformer, which can be used to guide the life assessment of transformer. The main component of insulating paper is the cellulose, which is made up of many units. The average number of cellulose units contained in a chain is called the Degree of Polymerization (DP) of the insulation paper. The aging process of insulating paper is closely related to the shortening process of the molecular chain, that is, the DP value decreasing process [12]. A first order model of transformer paper degradation depicted in (1)-(3) was introduced to describe the aging process of insulation paper.

$$
\begin{gathered}
d D P(t) / d t=-k(t)[D P(t)]^{2} \\
D P(t)=\frac{D P\left(t_{0}\right)}{1+D P\left(t_{0}\right) \int_{t_{0}}^{t} k(\tau) d \tau} \\
k(t)=A \exp \left(\frac{E_{a}}{R_{g} T(t)}\right)
\end{gathered}
$$

where $D P(t)$ is the $D P$ value at time $t, D P\left(t_{0}\right)$ is initial value of $D P$; $E_{a}$ is the initial aging activation energy of insulating paper; $A$ is the gas constant; $T(t)$ is the absolute temperature varying with time, which is set to be $370 \mathrm{~K}$ in this paper.

When $E_{a}=110.942 \mathrm{~kJ} / \mathrm{mol}, R_{g}=8.314, D P\left(t_{0}\right)=1000, A=2 \times 10^{8}$, and $T=$ $370 \mathrm{~K}$, the ideal DP curve changes with time is shown in Figure 1.

As depicted in Figure 1, it can be seen that with the increasing of the operation time, the insulation performance of the transformer is decreased, which may lead to equipment failure and cause power interruption. Actually, the insulation strength of the internal insulation of the transformer should be greater than the joint stress. When the insulation strength of the insulation paper is

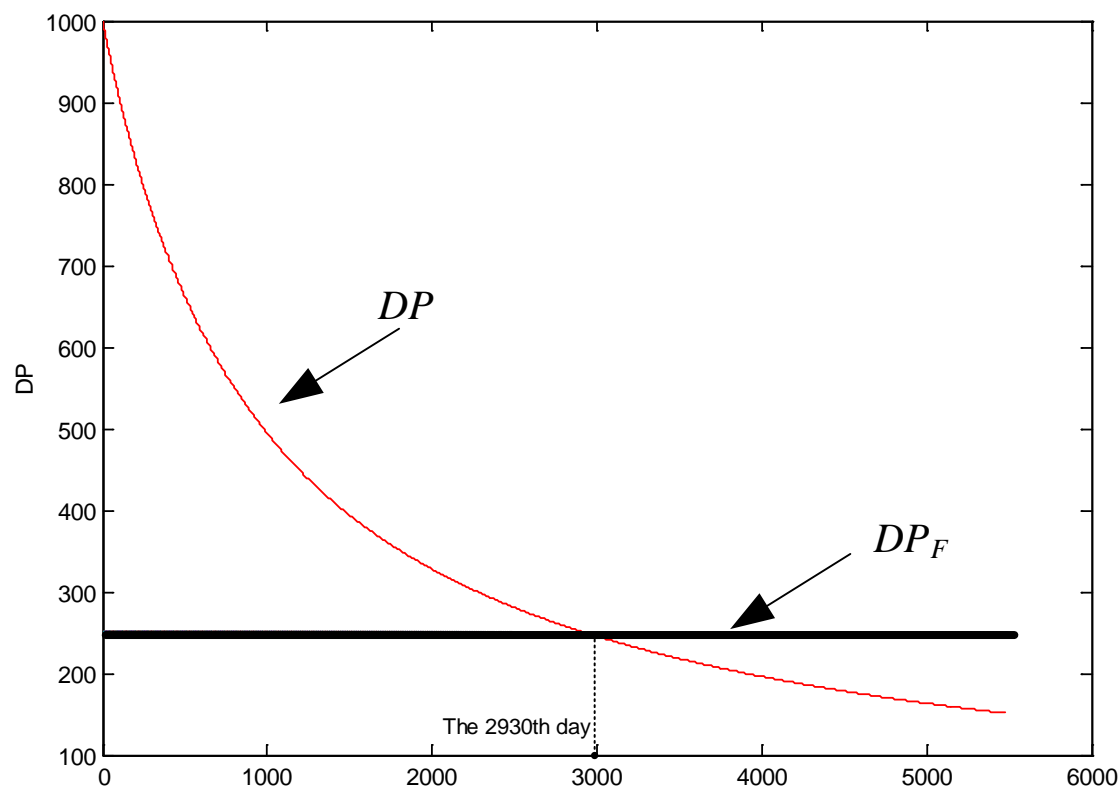

Figure 1. DP curve with time ideally. 
lower than the joint stress $\left(D P_{F}\right)$, the breakdown of the insulation paper will lead to the failure. At the same time, in the actual operation process, the life of the transformer will face a variety of uncertainties (also called unexpected stress), such as electrical stress, mechanical stress, thermal stress and chemical stress, which will significantly impact the insulation strength, just as shown in Figure 2.

It can be seen that under the normal condition, the insulation strength decreases with time. When the insulation strength is equal to the normal stress, the service life of the transformer is terminated. When suffered from some unexpected stresses, the insulation strength of the transformer is significantly reduced, which may lead to the reduction of the service life. Therefore, it is necessary to simulate the random effects of the unexpected stress on the insulation strength for replacement of old transformers.

\section{Replacement Decision Method Based on Probability Assessment of Failure Rate}

\subsection{Simulation of Transformer State Using Weibull Distribution}

The Weibull distribution is widely used to reflect the equipment failure rate changes with service age [13] [14]. By changing the shape parameter and scale parameter, it can be used to depicted the equipment failure rate tendency at different operation periods. For this reason, the Weibull distribution depicted in (4) is used to randomly generate the deviation value of DP $(\Delta D)$ when the unexpected stress happens.

$$
R=\exp \left[-\left(\frac{t}{\alpha}\right)^{\beta}\right]
$$

where $\alpha$ is the scale parameter; $\beta$ is the shape parameter. When $\beta>1$, it means the transformer is at the aging stage [15]. We assume that the operation data of transformer DP, can be obtained by on-line monitoring sensors. Thus, the difference between the DP value at a specific time and the DP expected value, which is depicted using $\Delta D$, is used as the statistical data for assessment. In the real application, the statistical data of $\Delta D$ is fitted by the

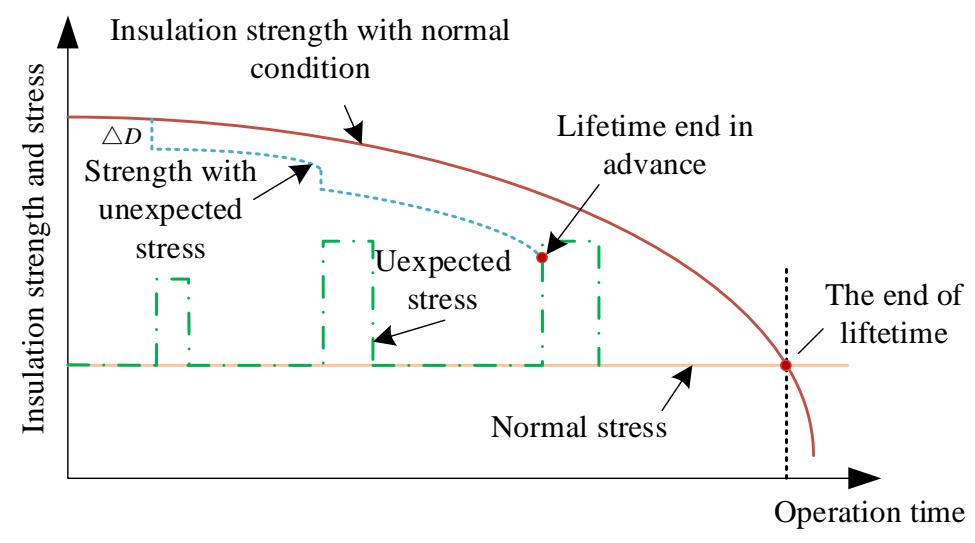

Figure 2. The comparison results of insulation strength between the normal and unexpected stress conditions. 
Weibull distributionin (4). Then the fitted Weibull distribution function is used by MCS to generate $\Delta D$ a teach specific time.

\subsection{Replacement Decision Method}

The steps of the proposed replacement decision method based on probability assessment of failure rate are given as follows:

Step 1: Set the Monte Carlo simulation times as $N$. The iteration time icount $=$ 1. The initial prediction time is $t_{0}$. The aim is to predict the future service life of the transformer within $n$ days. The simulation changing step is set to be 1 day. The transformer fault statistical vector is given in (5)

$$
\text { inum }=\left[\text { inum }_{t 0}, \text { inum }_{t 0+1}, \ldots, \text { inum }_{t i}, \ldots, \text { inum }_{n}\right]
$$

where $n$ is the forecast time period; inum $_{t i}=0$, when $t_{i}=t_{0},\left(t_{0} \leq t_{i} \leq n\right)$.

Step 2: The transformer statistical data of $\Delta D$ is fitted by the Weibull distribution with the corresponding scale and shape parameters using (4) at time $t_{i}$.

Step 3: The fitted Weibull distribution at time $t_{i}$ is used to generate $\Delta D_{t i}$ (the $\Delta D$ for iteration time icount at time $t_{i}$ ).

Step 4: Using the value $D_{t i}$ in Figure 1 at time $t_{i p}$ the DP value at time $t_{i}$ in the icount interation $\left(D P_{t i}=D_{t i}-\Delta D_{t i}\right)$ is determined. If $D P_{t i} \leq D P_{F}$ The expected service life $\left(t_{f}\right)$ of the transformer is obtained $\left(t_{f}=t_{i}\right)$, and the corresponding failure times at $t_{p}$ inum $_{t i}=$ inum $_{t i}+1$. Then, renew the iteration time icount $=$ icount +1 . Go to step 5. Otherwise, $t_{i}=t_{i}+\Delta t$, and go to step 2 .

Step 5: if icount $\leq N$, go to step 2. Otherwise, go to step 6 .

Step 6: According to inum, the fault ratecurve of the transformer changing with time is determined. For a specific time $t_{p}$ the fault rate $p_{\mathrm{f}_{-} t i}=i \operatorname{inum} m_{t i} / N$, which is used to determine the service life of the transformer.

Step 7: Set the failure probability threshold $\left(p_{f c}\right)$ for determining the service life of transformer. If $p_{\mathrm{f}_{-} t i} \geq p_{f o}$ the transformer is considered to be replaced and reaches the termination of its service life.

\subsection{Case Studies}

In order to verify the validity of the method proposed in this paper, a distribution transformer in a power supply company was used as a test case, and the simulation parameters are shown in Table 1 .

Table 1. Simulation parameters.

\begin{tabular}{cc}
\hline Parameters & Value \\
\hline$N$ & 50,000 \\
$E_{a}$ & $110.94 \mathrm{~kJ} / \mathrm{mol}$ \\
$R_{g}$ & 8.314 \\
$T$ & $370 \mathrm{~K}$ \\
$D P\left(t_{0}\right)$ & 1000 \\
$A$ & $2 \times 10^{8}$ \\
$D P_{F}$ & 250 \\
\hline
\end{tabular}


The comparison results of DP variation between the normal and unexpected stress conditions are given in Figure 3. Compared with Figure 1, for a specific simulation, the $D P$ decreases significantly with time under unexpected stress condition. The service life of transformer drops to 1979 days from 2945 days, when $D P_{F}=250$ days.

According to the method proposed in this paper, the failure rate in the last 25 days before the end of service life was analyzed. As seen in Figure 4, in the last

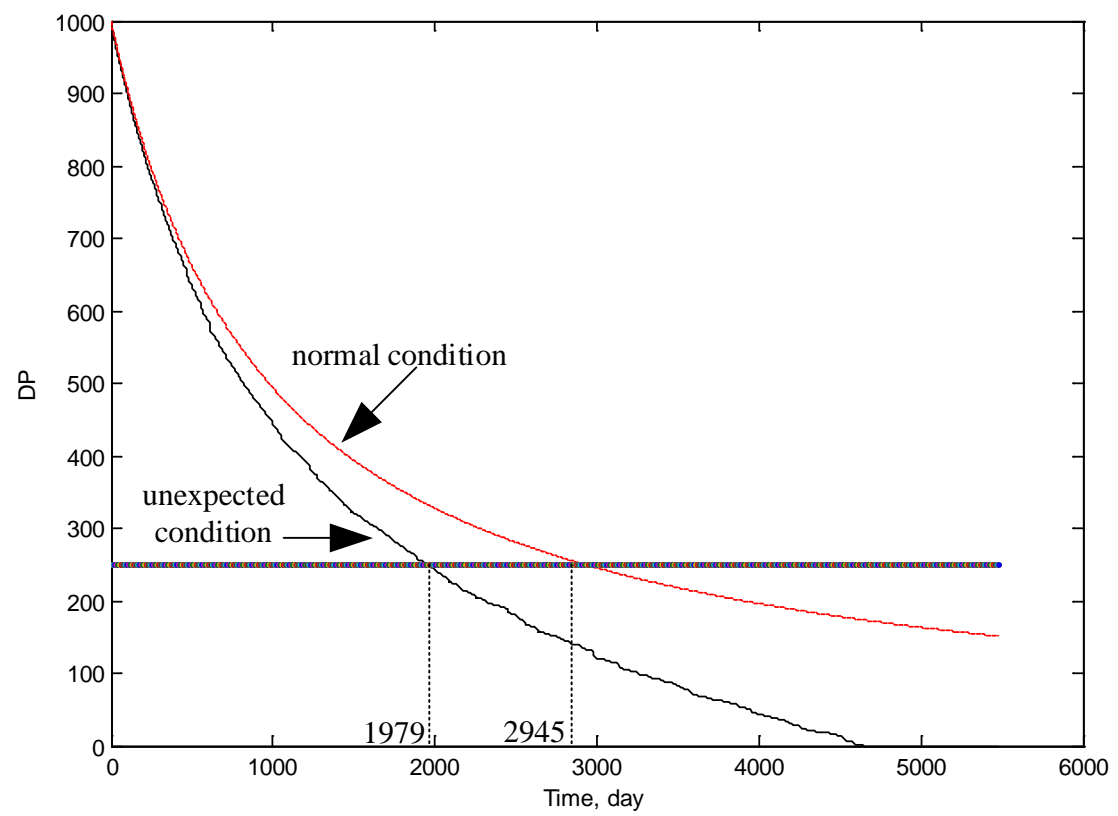

Figure 3. The comparison results of $D P$ variation between the normal and unexpected stress conditions.

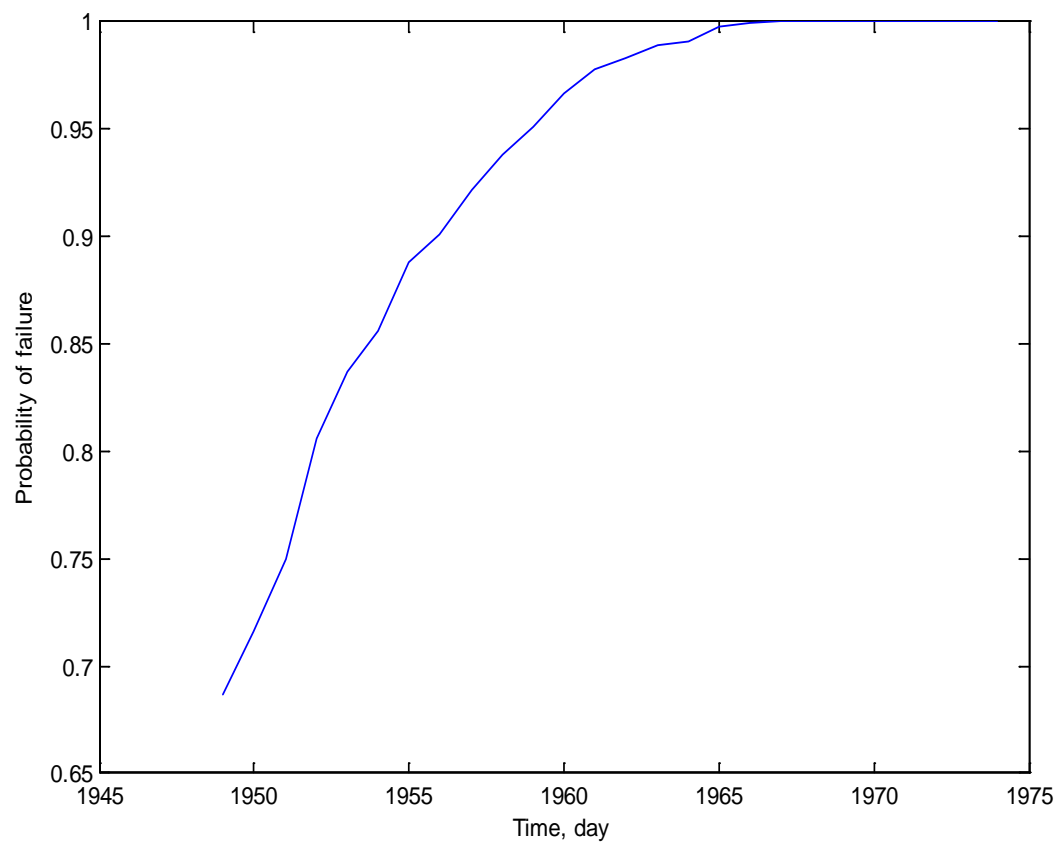

Figure 4. Failure rate before service life termination of a transformer. 
25 days, the failure rate of the transformer is continuously increasing. At the end of day 1979 , the failure rate is gradually approaching to $100 \%$. It can be considered that the service life of the transformer is terminated, and the transformer should be replaced.

\section{Conclusion}

The service life of a transformer is significantly influenced by unexpected stresses. As a result, it is of great practical value to put forward a decision method for transformer replacement, in order to guide the transformer technical transformation process. For this reason, a transformer replacement decision method based on probability assessment of FR is proposed. A first order model of transformer paper degradation is firstly proposed. The Weibull Distribution is used by MCS to generate the variations of $D P$ along time based on historical data, and the transformer FR is determined. When the FR is higher than a pre-defined threshold value, the transformer should be replaced for reliability purpose. Case studies show that the method can effectively identify the influences of various uncertainties on the service life of transformer, and improve the technical level and economic benefit of power company, which has a good engineering application prospect.

\section{Acknowledgements}

This work was supported by the project: Research on key techniques to the design of smart substation in Tianjin (KJ15-1-08).

\section{References}

[1] Aldhubaib, H.A. and Salama, M.M.A. (2014) A Novel Approach to Investigate the Effect of Maintenance on the Replacement Time for Transformers. IEEE Transactions on Power Delivery, 42, 291-293. https://doi.org/10.1109/tpwrd.2014.2321409

[2] Zhou, D., Wang, Z.D., Jarman, P. and Li, C.R. (2014) Data Requisites for Transformer Statistical Lifetime Modelling-Part II: Combination of Random and AgingRelated Failures. IEEE Transactions on Power Delivery, 29, 154-160. https://doi.org/10.1109/TPWRD.2013.2270116

[3] Zhuang, Q., Morshuis, P.H.F., Chen, X. and Smit, J.J. (2012) Life Prediction of a Full-Scale Transformer Winding Insulation through Statistical Analysis of AC Voltage Endurance Test Data. IEEE Transactions on Dielectrics and Electrical Insulation, 19, 460-471. https://doi.org/10.1109/TDEI.2012.6180239

[4] Adoghe, A.U., Awosope, C.O.A. and Daramola, S.A. (2010) Modeling Distribution Component Deterioration: An application to Transformer Insulation. Advanced Materials Research, 367, 117-123. https://doi.org/10.4028/www.scientific.net/AMR.367.117

[5] Shi, J., Wu, C.N. and Wang, X.J. (2016) Life Evaluation of Insulation Paper Used in Transformer by Determining the Activation Energy. Material Research Innovations, 19, 164-167.

[6] Yang, Z.C., Zhang, C.L., Wu, Y., et al. (2014) Research on Rough Sets and RBF Neural Network Based Transformer Fault Diagnosis Method. Electrical Measurement \& Instrumentation, 51, 34-39. 
[7] Zhang, K., Yuan, F., Guo, J. and Wang, G. (2016) A Novel Neural Network Approach to Transformer Fault Diagnosis Based on Momentum-Embedded BP Neural Network Optimized by Genetic Algorithm and Fuzzy C-Means. Arabian Journal for Science and Engineering, 9, 1-11. https://doi.org/10.1016/j.msea.2016.04.010

[8] Liao, R., Bian, J., Yang, L. and Grzybowski, S. (2013) Cloud Model-Based Failure Mode and Effects Analysis for Prioritization of Failures of Power Transformer in Risk Assessment. International Transactions on Electrical Energy Systems, 23, 11721190.

[9] Sun, Y.Y., Wang, X., Zheng, Y.H., Li, L.X. and Xu, Q.S. (2014) Power Transformer Life Analysis Based on Risk Assessment. Applied Mechanics \& Materials, 672-674, 1151-1154. https://doi.org/10.4028/www.scientific.net/AMM.651-653.1151

[10] Guo, Y.J., Dai, Y.J. and Xi, Y.J. (1996) A New Methodology for Lifetime Evaluation of Transformer: Part I. Theory of Lifetime Evaluation. Proceedings of ICEE 96, Beijing.

[11] Pugh, P.F., Bernstein, B.S. and Brancato, E.L. (1994) Aging of Equipment in the Electric Utilities. IEEE Transactions on Dielectrics \& Electrical Insulation, 1, 164165. https://doi.org/10.1109/94.300246

[12] Guo, Y.J. (2001) New Methodology for Lifetime Evaluation of Medium and Small Power Transformer. Automation of Electric Power Systems, 25, 38-41.

[13] Jiang, P., Lim, J.H., Zuo, M.J. and Guo, B. (2010) Reliability Estimation in a Weibull Lifetime Distribution with Zero-Failure Field Data. Quality and Reliability Engineering International, 26, 691-701. https://doi.org/10.1002/qre.1138

[14] Cheng, Y.F. and Sheu, S.H. (2016) Robust Estimation for Weibull Distribution in Partially Accelerated Life Tests with Early Failures. Quality and Reliability Engineering International, 32, 2207-2216. https://doi.org/10.1002/qre.1928

[15] Qin, M., Wu, S., Peng, X., et al. (2008) A Reliability Evaluation Method for Equipment with a Weibull Failure Distribution. Engineering Journal of Wuhan Universi$t y, 41,100-102$.

\section{Scientific Research Publishing}

\section{Submit or recommend next manuscript to SCIRP and we will provide best} service for you:

Accepting pre-submission inquiries through Email, Facebook, LinkedIn, Twitter, etc. A wide selection of journals (inclusive of 9 subjects, more than 200 journals)

Providing 24-hour high-quality service

User-friendly online submission system

Fair and swift peer-review system

Efficient typesetting and proofreading procedure

Display of the result of downloads and visits, as well as the number of cited articles

Maximum dissemination of your research work

Submit your manuscript at: http://papersubmission.scirp.org/

Or contact epe@scirp.org 